\title{
Exploitation of De Novo Helper-Lipids for Effective Gene Delivery
}

\author{
Tomoaki Kurosaki ${ }^{1}$, Takashi Kitahara ${ }^{1}$, Mugen Teshima ${ }^{1}$, Koyo Nishida ${ }^{2}$, Junzo Nakamura ${ }^{2}$, Mikiro \\ Nakashima ${ }^{2}$, Hideto To ${ }^{1}$, Hiromitsu Fukuchi ${ }^{1}$, Tomoyuki Hamamoto ${ }^{1}$, Hitoshi Sasaki ${ }^{1 *}$ \\ ${ }^{1}$ Department of Hospital Pharmacy, University Hospital of Medicine and Dentistry, 1-7-1 Sakamoto, Nagasaki \\ 852-8501, Japan; ${ }^{2}$ Course of Medical and Dental Sciences, Nagasaki University Graduate School of Biomedical \\ Sciences, 1-14 Bunkyo-machi, Nagasaki 852-8521, Japan.
}

Received April 1, 2008; Revised October 28, 2008; Accepted December 29, 2008, Published December 30, 2008.

\begin{abstract}
Purpose. In this experiment, we developed new cationic liposomes as a vector, which form the complex with plasmid DNA (pDNA), for gene delivery after systemic administration. We investigated penetration enhancers as an additional component of the cationic liposomes because of their fusogenic activities. Methods. Transdermal penetraion enhancers such as N-lauroylsarcosine (LS), (R)-(+)-limonene (LM), vitamin E (VE), and phosphatidyl choline from eggs (EggPC) were used in this experiments as helper-lipids with $\mathrm{N}-[1-(2$,
\end{abstract} 3-dioleyloxy)propyl]-N,N,N-trimethlylammonium chloride (DOTMA) and cholesterol (CHOL). We examined in vitro transfection efficiency, cytotoxicity, hematotoxicity, and in vivo transfection efficiency of pDNA/cationic liposomes complexes. Results. In transfection experiments in vitro, the cationic lipoplexes containing LS had highest transfection efficiency among the other lipoplexes independently of fetal bovine serum (FBS). Furthermore, the lipoplexes containing LS had lowest cell toxicity among the other lipoplexes in the presence of FBS. As the results of erythrocytes interaction experiment, DOTMA / LS / CHOL, DOTMA / VE / CHOL, and DOTMA / EggPC / CHOL lipoplexes showed extremely lower hematotoxicity. On the basis of these results, the in vivo transfection efficiencies of the lipoplexes were examined. The lipoplexes containing LS had the highest transfection activity among the lipoplexes. Conclusion. In conclusion, several transdermal penetration enhancers are available for the helper-lipids in cationic liposomal vectors. Among them, DOTMA/LS/CHOL lipoplexes showed superior characteristics in in vitro transfection efficiency, cell toxicity, hematotoxicity, and in vivo transfection efficiency.

\section{INTRODUCTION}

Gene therapy is a useful treatment for curing inborn and acquired diseases such as cancer, cardiovascular disease, and rheumatoid arthritis (1, 2). Nucleic acids, however, are rapidly degraded by nucleases and exhibit poor cellular uptake when delivered in aqueous solutions. The gene delivery vectors used so far include viral systems such as retroviruses, adenoviruses, and adeno-associated viruses or non-viral systems including cationic liposomes and polymers $(3,4)$.

Among non-viral systems, cationic lipsosomes have been reported to be suitable for effective human gene therapy (5-7). The complex of cationic liposomes with pDNA showed high gene expression in the liver and spleen after systemic administration. Cationic liposomes are constructed with cationic lipids and "helper-lipids". Cationic lipids, N-[1-(2, 3-dioleyloxy) propyl]-N, N, $\mathrm{N}$-trimethlylammonium chloride (DOTMA) are often used for forming electrostatic complexes with DNA (8). Cholesterol (CHOL) and dioleyl phosphatidylethanolamine (DOPE) are well known helper-lipids. CHOL was known to stabilize liposomal membrane, and they are often added to cationic liposomes to attain higher transfection levels in vivo $(9,10)$. DOPE is a fusogenic lipid to destabilize lipid bilayers by promoting the transition from a lamellar to a hexagonal phase, it is believed to be involved in endosomal disruption.

Corresponding Author: H Sasaki, Department of Hospital Pharmacy, University Hospital of Medicine and Dentistry, 1-7-1 Sakamoto, Nagasaki 852-8501, Japan. E-mail sasaki@nagasaki-u.ac.jp 
The component of fusogenic lipids is one of the important factors for determining the gene expression of cationic liposomal vectors $(11,12)$.

DOPE was also reported as efficient transdermal penetration enhancer of some drugs such as estradiol, progesterone, and propranolol (13). If so, the transdermal penetration enhancers may play a role of fusogenic lipids for gene delivery. Actually, the transdermal penetration enhancers were known as inserting to dermal cornified lipid layer, and they may be disposed to disrupt the lipid membrane $(14,15)$. According to this hypothesis, we investigated the effect of frequently used penetration enhancers as alternative helper-lipids to DOPE on gene delivery of cationic liposomes. N-Lauroylsarcosine (LS), (R)-(+)-limonene (LM), vitamin E (VE), and phosphatidyl choline from eggs (EggPC) were used in the experiments as penetration enhancers.

\section{METHODS}

\section{Chemicals}

DOTMA was obtained from Tokyo Chemical Industry Co. (Tokyo, Japan). LS and LM were obtained from Aldrich Chemical Co. (Milwaukee, WI, USA). CHOL and VE were obtained from Nacalai Tesque, Inc. (Kyoto, Japan). DOPE, EggPC (COATSOME NC-10S) was purchased from Nippon Oil and Fats Co. (Tokyo, Japan). Fetal bovine serum (FBS) was obtained from Biosource International Inc. (Camarillo, CA, USA). Dulbecco's modified Eagle medium (DMEM), antibiotics (penicillin $100 \mathrm{U} / \mathrm{mL}$ and streptomycin $100 \mu \mathrm{g} / \mathrm{mL}$ ), and other culture reagents were obtained from GIBCO BRL (Grand Island, NY, USA). 2-(4-lodophenyl)-3-(4-nitrophenyl)-5-(2, 4-disulfophenyl)-2H-tetrazolium, monosodium salt (WST-1) and 1-methoxy-5-methylphenazinium methylsulfate (1-methoxy PMS) were obtained from Dojindo Laboratories (Kumamoto, Japan). All other chemicals were of the highest purity available.

Construction and Preparation of pDNA (pCMV-Luc)

pCMV-Luc was constructed by subcloning the Hind III/Xba I firefly luciferase cDNA fragment from
pGL3-control vector (Promega, Madison, WI, USA) into the polylinker of pcDNA3 vector (Invitrogen Corp., Carlsbad, CA, USA). The pDNA was amplified in the E. coli strain XL1-blue, isolated, and purified using an EndoFree QIAGEN Plasmid Giga Kit (QIAGEN GmbH, Hilden, Germany). Purified pDNA was diluted in the sterile $5 \%(\mathrm{w} / \mathrm{v})$ glucose and stored at $-80{ }^{\circ} \mathrm{C}$ until use. The concentration of pDNA was measured by at $260 \mathrm{~nm}$ and it was adjusted to $1 \mathrm{mg} / \mathrm{mL}$.

\section{Preparation of Cationic Liposomes}

DOTMA/DOPE/CHOL (1:0.5:0.5 molar ratio), DOTMA/LS/CHOL (1:0.5:0.5 molar ratio), DOTMA/LM/CHOL (1:0.5:0.5 molar ratio), DOTMA/VE/CHOL (1:0.5:0.5 molar ratio), and DOTMA/EggPC/CHOL (1:0.5:0.5 molar ratio) liposomes were prepared according to a previous report (16). The lipids were dissolved in chloroform. Mixtures of each lipid were dried as a thin film in a test tube using an evaporator at 25 ${ }^{\circ} \mathrm{C}$, and then were vacuum-desiccated for approximately $4 \mathrm{~h}$. The film was resuspended in sterile 5\% dextrose. After hydration, the dispersions were sonicated at $100 \mathrm{~W}$ for $3 \mathrm{~min}$ on ice. The resulting liposomes were extruded 11 times through doublestacked $100 \mathrm{~nm}$ polycarbonate membrane filters.

\section{Preparation of pDNA/Cationic Liposome Complexes}

First, the mixing ratio of liposomes and pDNA was expressed as a +/- charge ratio, which is the molar ratio of DOTMA to pDNA phosphate residue. For gene expression experiments, the appropriate amounts of the cationic liposome solution were mixed with the pDNA solution as +1.75 charge ratio, and mixed thoroughly by pipetting and left for $30 \mathrm{~min}$ at room temperature to allow complex formation. The particle size and $\zeta$-potential of the liposomes were measured by use of the $\zeta$-sizer Nano-ZS (Malvern Instruments, Ltd., Worcestershire, UK). The number-fractioned mean diameter was shown.

\section{Electrophoresis Assay}

Each lipoplexes containing $1 \mu \mathrm{g}$ of pDNA and appropriate amounts of the liposomes was prepared 
as total volume $10 \mu \mathrm{L}$. The $5 \mu \mathrm{L}$ of each lipoplexes solution were mixed with $1 \mu \mathrm{L}$ loading buffer ( $30 \%$ glycerol and $0.2 \%$ bromophenol blue) and were loaded onto a $0.8 \%$ agarose gel containing $0.03 \%$ ethidium bromide. Electrophoresis (i-Mupid J, Cosmo Bio, Tokyo, Japan) was carried out with a current $50 \mathrm{~V}$ in running buffer solution (40 mM Tris- $\mathrm{HCl}, 40 \mathrm{mM}$ acetic acid, and $1 \mathrm{mM}$ EDTA buffer). The retardations of the pDNA were visualized by use of the FluorChem Imaging Systems (Alpha Innotech, CA, USA).

\section{In Vitro Gene Expression Experiments}

The human hepatoma cell line HepG2 was obtained from Cell Resource Center for Biomedical Research, Tohoku University. The HepG2 cells were maintained in the FBS $(+)$ medium (DMEM supplemented with $10 \%$ FBS and antibiotics) or the FBS (-) medium (DMEM supplemented with antibiotics) at $37{ }^{\circ} \mathrm{C}$ under an atmosphere of $5 \%$ $\mathrm{CO}_{2}$ in air. The cells were plated on biocoat collagen I cellware 12-well collagen-coating plates (Becton-Dickinson, Franklin Lakes, NJ, USA) at a density of $5 \times 10^{4}$ cells/well in the FBS $(+)$ medium. After $24 \mathrm{~h}$, the medium was replaced with the FBS $(+)$ medium or the FBS (-) medium, containing 2 $\mu \mathrm{g} / \mathrm{mL}$ pDNA complexed with various cationic liposomes for $6 \mathrm{~h}$. After transfection, the medium was replaced again with the FBS $(+)$ medium, and then incubated for $24 \mathrm{~h}$. The cells were washed with phosphate-buffered saline (PBS) twice, and then lysed in $200 \mu \mathrm{L}$ of lysis buffer $(0.05 \%$ Triton $\mathrm{X}-100,2 \mathrm{mM}$ EDTA, and $0.1 \mathrm{M}$ Tris/ $\mathrm{HCl}, \mathrm{pH}=$ 7.8). The $10 \mu \mathrm{L}$ lysate was mixed with $50 \mu \mathrm{L}$ luciferase assay buffer (Picagene, Toyo Ink, Tokyo, Japan) and the light produced was immediately measured using a luminometer (Lumat LB 9507, EG \& G Berthold, Bad Wildbad, Germany). The protein content of the lysate was determined by a Bradford assay using BSA as a standard. The absorbance was measured using a microplate reader (Spectra 1, TECAN Austria Gmb.H., Grodig, Austria) at $570 \mathrm{~nm}$. The luciferase activity is indicated as the relative number of light units (RLU) per mg protein. The result in a well was counted as one experiment.

\section{WST-1 Assay}

Cytotoxicity tests on HepG2 cells were carried out using a WST-1 assay. The WST- 1 reagent was prepared (5mM WST-1 and $0.2 \mathrm{mM} 1$-methoxy PMS in PBS) and was filtered through a $0.22 \mu \mathrm{m}$ filter (Millex-GP, Millipore Co, Bedford, MA, USA) prior to use. HepG2 cells were plated on 96-well plates (Becton-Dickinson, Franklin Lakes, NJ, USA) at a density of $5 \times 10^{3}$ cells/well in the FBS (+) medium. After transfection as the method above-mentioned, the medium was replaced again with $100 \mu \mathrm{L}$ FBS $(+)$ medium and $10 \mu \mathrm{L}$ of the WST-1 reagent was added to each well. The wells were incubated for an additional $2 \mathrm{~h}$ at 37 ${ }^{\circ} \mathrm{C}$ and then $10 \mu \mathrm{L}$ of $0.1 \mathrm{~N} \mathrm{HCl}$ was added to each well. The absorbance was measured using a microplate reader at $450 \mathrm{~nm}$ (reference wavelength $=650 \mathrm{~nm}$ ). Untreated cells used as $100 \%$ viability.

\section{Animals}

Animal care and experimental procedures were performed in accordance with the Guidelines for Animal Experimentation of Nagasaki University with approval of the Institutional Animal Care and Use Committee. Male ddY mice (5-6 weeks old) were purchased from Japan SLC (Shizuoka, Japan). After shipping, mice were acclimatized to the environment for one day at least before experiments were begun.

\section{Interaction with Erythrocytes}

Erythrocytes from mice were washed three times at $4{ }^{\circ} \mathrm{C}$ by centrifugation at 3,000 rpm (Kubota 3700, Kubota, Tokyo, Japan) for $15 \mathrm{~min}$ and resuspend in PBS. For agglutination study, 2\% stock suspension was prepared. The various lipoplexes were added to the erythrocytes. The suspensions were incubated for $15 \mathrm{~min}$ at room temperature (RT). The $10 \mu \mathrm{L}$ suspensions were put on a glass plate and agglutination was observed by optical microscopy (400× magnifications). For hemolysis study, 5\% stock suspension was prepared. The various lipoplexes were added to the erythrocytes and the suspensions were incubated $24 \mathrm{~h}$ at RT. After incubation, the suspensions were centrifuged at 3,000 rpm for $15 \mathrm{~min}$, and supernatants were taken. Hemolysis was quantified by measuring 
the hemoglobin release at $545 \mathrm{~nm}$. The lysis buffer was used for $100 \%$ hemolysis sample.

\section{In Vivo Gene Expression Experiments}

The lipoplexes were prepared by mixing the $40 \mu \mathrm{g}$ of pDNA and appropriate amounts of the cationic liposomes before every experiment. Six mice were administrated intravenously with DOTMA/DOPE/CHOL and DOTMA/LS/CHOL lipoplexes, and three mice were administrated intravenously with DOTMA/LM/CHOL, DOTMA/VE/CHOL, and DOTMA/EggPC/CHOL lipoplexes. After $6 \mathrm{~h}$ following administration, mice were sacrificed and the liver, kidney, spleen, heart, and lung were dissected. The tissues were washed twice with cold saline and homogenized with the lysis buffer. The lysis buffer was added in a weight ratio of $3 \mu \mathrm{L} / \mathrm{mg}$ for liver samples, 5 $\mu \mathrm{L} / \mathrm{mg}$ for kidney samples, and $10 \mu \mathrm{L} / \mathrm{mg}$ for other organ samples. The homogenates were centrifuged at $15,000 \mathrm{rpm}$ for $5 \mathrm{~min}$. The supernatants were used for luciferase assays. The $10 \mu \mathrm{L}$ of supernatant was mixed with $50 \mu \mathrm{L}$ of luciferase assay buffer and the light produced was immediately measured using the luminometer. The luciferase activity is indicated as RLU per gram of tissue.

\section{Statistical Analysis}

Multiple comparisons test was performed by Dunnett's pairwise multiple comparison $t$ test.

\section{RESULTS}

\section{Physicochemical Characteristics}

The particle sizes and $\zeta$-potentials of the liposomes containing those penetration enhancer lipids were measured (Table 1). DOTMA/DOPE/CHOL liposomes showed $19.30 \pm 5.35 \mathrm{~nm}$ of particle sizes and $+67.07 \pm 1.01 \mathrm{mV}$ of $\zeta$-potentials. The particle sizes of DOTMA/LS/CHOL and DOTMA/VE/CHOL liposomes were significantly larger than DOTMA/DOPE/CHOL liposomes $(P<$ 0.01 ), furthermore, $\zeta$-potentials of the liposomes with the lipids were significantly lower than the DOTMA/DOPE/CHOL liposomes $(P<0.01)$.

\section{Electrophoresis Assay}

Electrophoresis assay was used to confirm the strong formations of the lipoplexes. By mixing the liposomes and pDNA solution, the lipoplexes were formed as evidenced by agarose gel electrophoresis (Figure 1). In the lane of naked pDNA (lane 1), migration of pDNA was not retarded, and the pDNA bands were clearly observed. On the other hand in the lane of each lipoplexes (lane 2-6), migrations of pDNA were retarded and the pDNA bands were not observed.

\section{In Vitro Transfection Activities}

For in vitro transfection experiments, the cationic liposomes were complexed with pDNA to form the lipoplexes. In the preliminary experiment, +1.75 charge ratio lipoplexes showed the highest luciferase activity in the HepG2 cells among +1.5 , $+1.75,+2$ charge ratio. Then, the each lipoplexes at +1.75 charge ratio was added to HepG2 cells in FBS (-) or FBS $(+)$ medium and the luciferase activities were evaluated (Figures $2 \mathrm{~A}$ and $2 \mathrm{~B}$ ). Among them, DOTMA/LS/CHOL lipoplexes showed the highest luciferase activity in FBS (-) medium. The activity of DOTMA/LS/CHOL lipoplexes was significantly higher than that of the DOTMA/DOPE/CHOL lipoplexes $(P<0.01)$. On the other hand, all lipoplexes showed high luciferase activity in FBS $(+)$ medium.

\section{In Vitro Cellular Toxicities}

The cell viabilities were evaluated by WST-1 assay, and each lipoplexes was added to HepG2 cells in FBS (-) or FBS (+) medium (Figures 3A and 3B). The cell viabilities of all lipoplexes were significantly lower than the control in FBS (-) medium $(P<0.01)$. The DOTMA/VE/CHOL lipoplexes showed markedly higher viability than DOTMA/DOPE/CHOL. On the other hand, DOTMA/LS/CHOL showed lowest cytotoxicity in FBS (+) medium. The cell viabilities of all lipoplexes containing the penetration enhancer lipids were higher than that of DOTMA/DOPE/CHOL lipoplexes. 
Table 1. Physicochemical characteristics of the liposomes.

\begin{tabular}{lll} 
Lipid composition $(1 / 0.5 / 0.5)$ & Size $(\mathrm{nm})$ & $\zeta$-potential $(\mathrm{mV})$ \\
\hline DOTMA/DOPE/CHOL & $19.80 \pm 5.35$ & $+67.07 \pm 1.01$ \\
DOTMA/LS/CHOL & $225.00 \pm 22.34^{\mathrm{a}}$ & $+22.77 \pm 0.71^{\mathrm{a}}$ \\
DOTMA/LM/CHOL & $44.60 \pm 2.93$ & $+45.53 \pm 0.15^{\mathrm{a}}$ \\
DOTMA/VE/CHOL & $92.77 \pm 12.94^{\mathrm{a}}$ & $+45.70 \pm 0.60^{\mathrm{a}}$ \\
DOTMA/EggPC/CHOL & $23.57 \pm 11.83$ & $+51.83 \pm 1.89^{\mathrm{a}}$ \\
\hline
\end{tabular}

Each value represents the mean \pm S.D. of three experiments.

${ }^{\mathrm{a}}: P<0.01$ vs DOTMA/DOPE/CHOL.

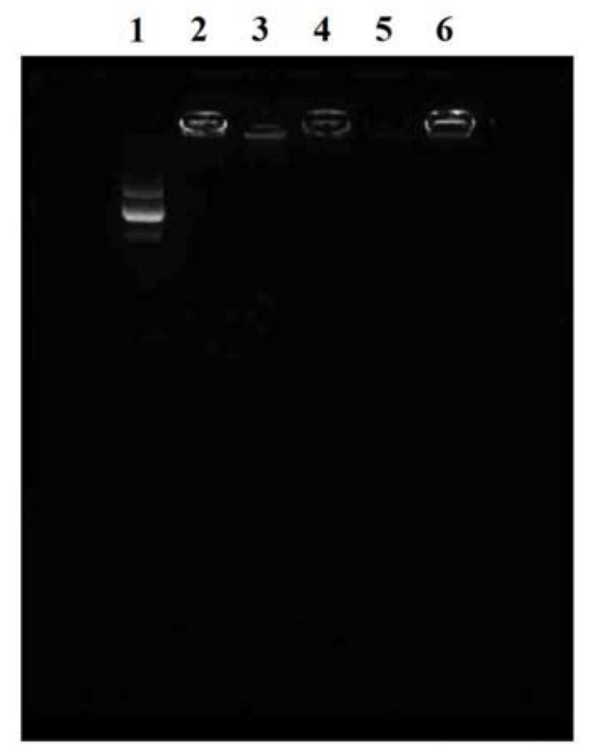

Figure 1. Effect of each liposome on electrophoretic migration of pDNA through an agarose gel. 1: naked pDNA; 2: DOTMA/DOPE/CHOL lipoplexes; 3: DOTMA/LS/CHOL lipoplexes; 4: DOTMA/LM/CHOL lipoplexes; 5: DOTMA/VE/CHOL lipoplexes; 6: DOTMA/EggPC/CHOL lipoplexes.
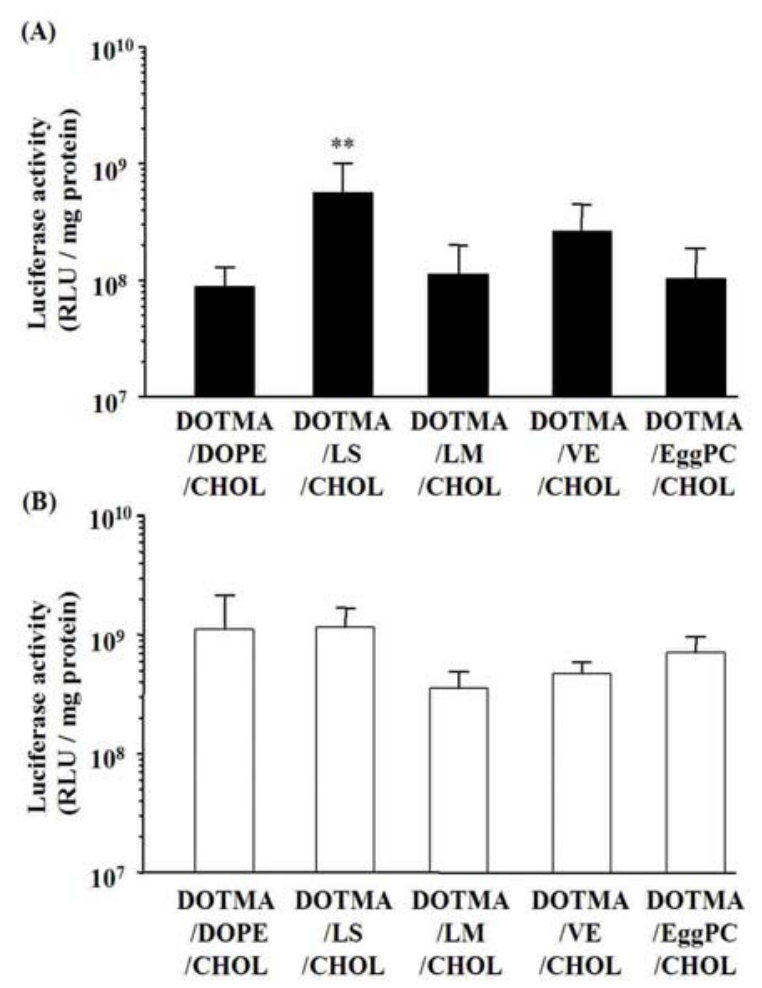

Figure 2. Luciferase gene expression of the various lipoplexes in vitro. The lipoplexes were added to the HepG2 cells in the FBS (-) medium (A) or FBS (+) medium (B). After $24 \mathrm{~h}$, cells were lysed and luciferase activities were determined. Each bar represents the mean + S.D. of seven experiments. **; $P<0.01$ vs DOTMA/DOPE/CHOL lipoplexes. 


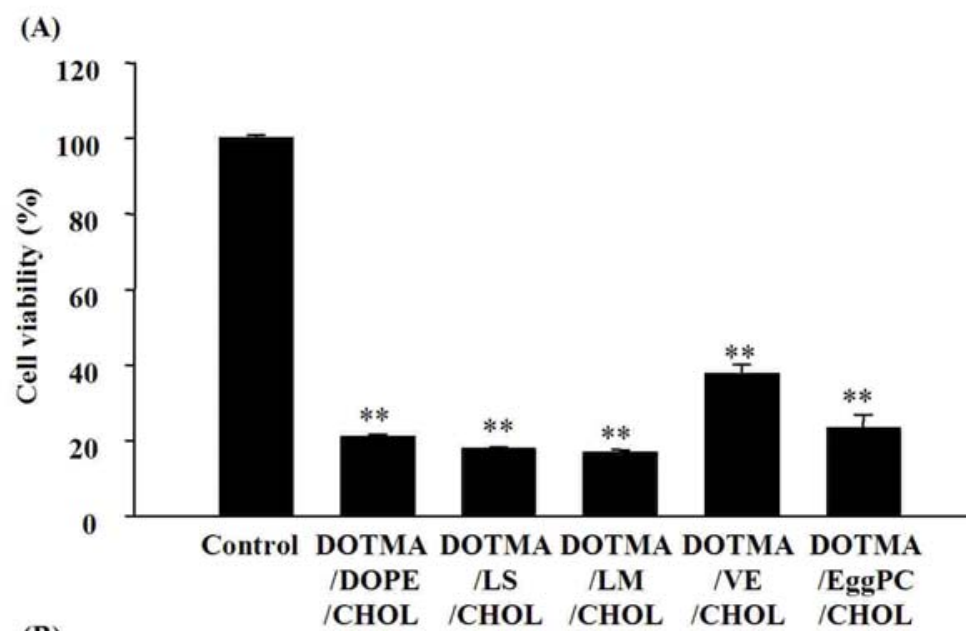

(B)

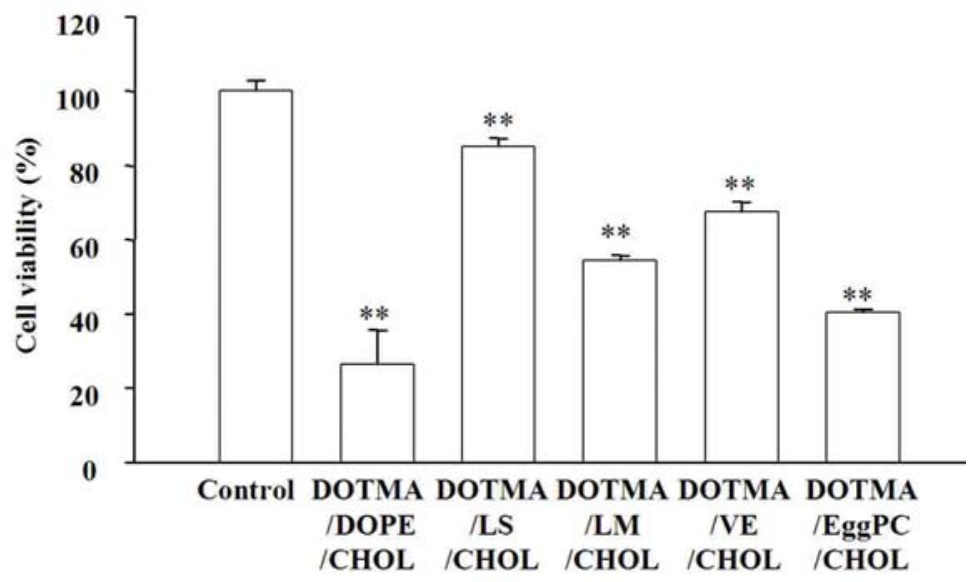

Figure. 3. Cell viabilities of various lipoplexes. The lipoplexes were added to the HepG2 cells in the FBS (-) medium (A) or FBS $(+)$ medium (B). The cell viabilities were determined by WST-1 assay. Each bar represents the mean + S.D. of three or four experiments. ${ }^{* *} ; P<0.01$ vs control.

\section{Interaction with Erythrocytes}

The lipoplexes were added to erythrocytes to evaluate agglutination and hemolysis (Figures 4 and 5). DOTMA/DOPE/CHOL lipoplexes showed severe agglutination in microscopy but no agglutination was observed in other lipoplexes. Hemolysis was quantified by measuring the hemoglobin release.

DOTMA/LS/CHOL, DOTMA/VE/CHOL, and DOTMA/EggPC/CHOL lipoplexes had significantly lower hemolysis activities than DOTMA/DOPE/CHOL lipoplexes $(P<0.01)$. However, DOTMA/LM/CHOL lipoplexes had significantly higher hemolysis activities than
DOTMA/DOPE/CHOL lipoplexes $(P<0.01)$.

\section{In Vivo Transfection Activities}

For in vivo transfection experiments, the each lipoplexes was administrated intravenously to mice. After 6 hours, the five organs such as liver, kidney, spleen, heart, and lung were dissected and those luciferase activities were determined (Figure 6). In the all organs, DOTMA/LS/CHOL lipoplexes had highest transfection efficiency among the five lipoplexes. At the same time, they had significantly higher transfection efficiency than DOTMA/DOPE/CHOL lipoplexes in the liver, heart, and lung $(P<0.01)$. 

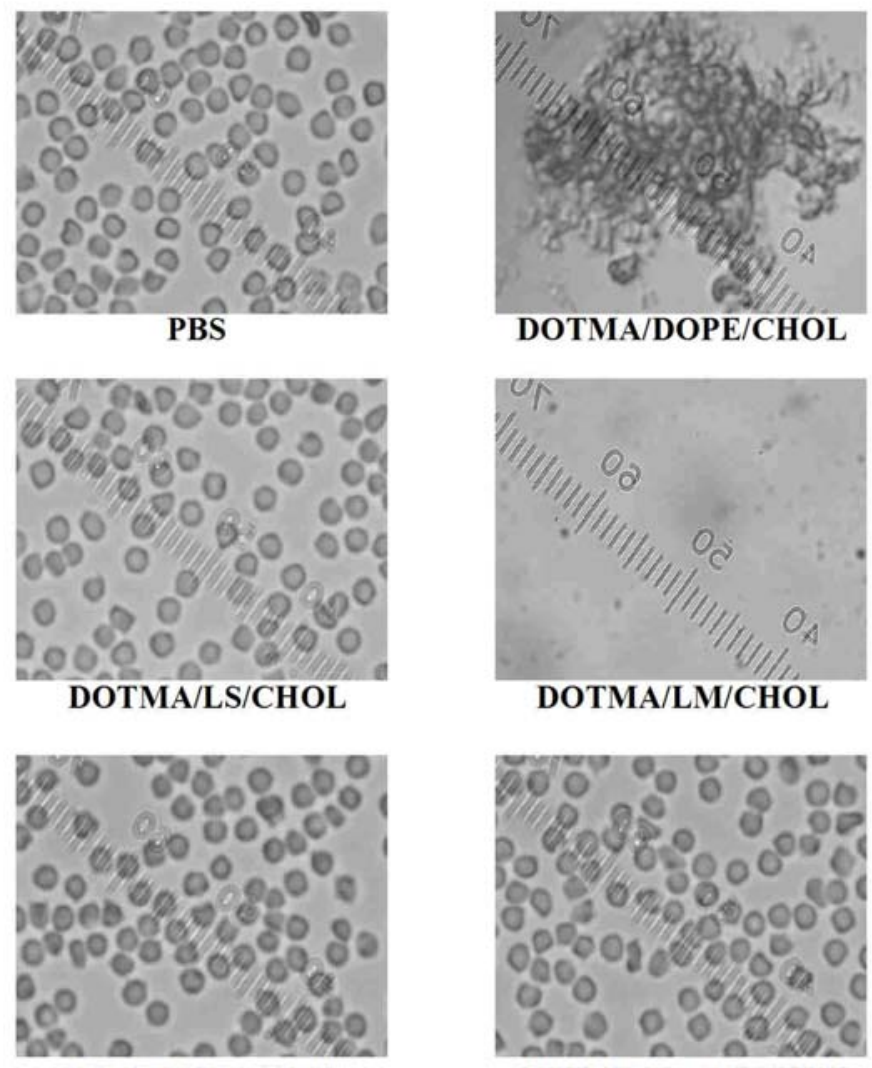

DOTMA/VE/CHOL

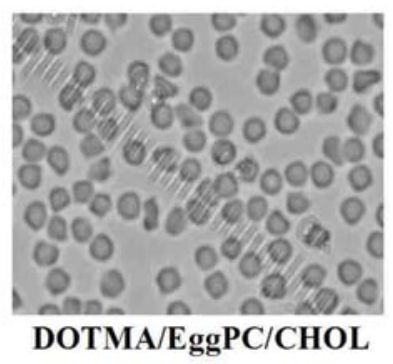

Figure 4. Agglutination of erythrocytes by lipoplexes. Each lipoplexes was added to erythrocytes, and agglutination was observed by phase microscopy (400× magnifications).

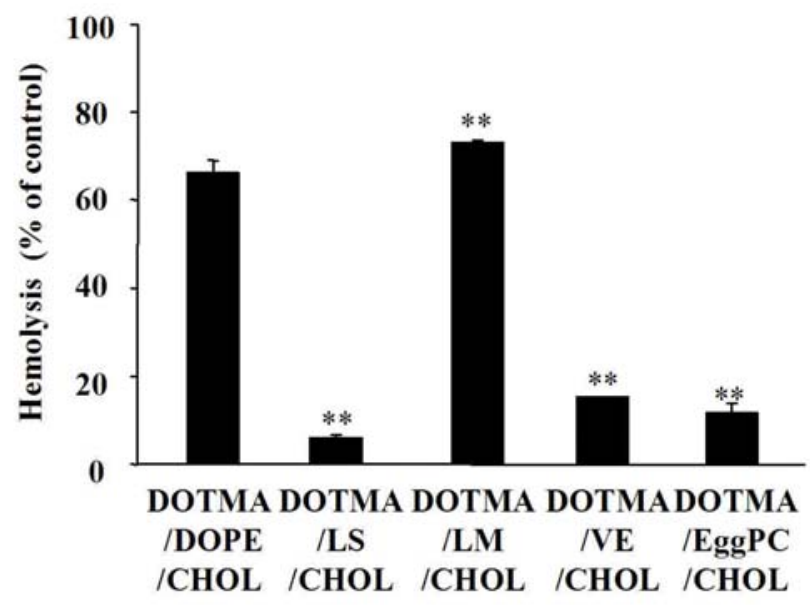

Figure 5. Hemolysis of erythrocytes by lipoplexes. Each lipoplexes was added to erythrocytes, and incubated $24 \mathrm{~h}$. The hemolysis activity was determined by measuring the hemoglobin release at $545 \mathrm{~nm}$. Each bar represents the mean + S.D. of three experiments. $\quad * * ; P<0.01$ vs DOTMA/DOPE/CHOL lipoplexes. 
(A)

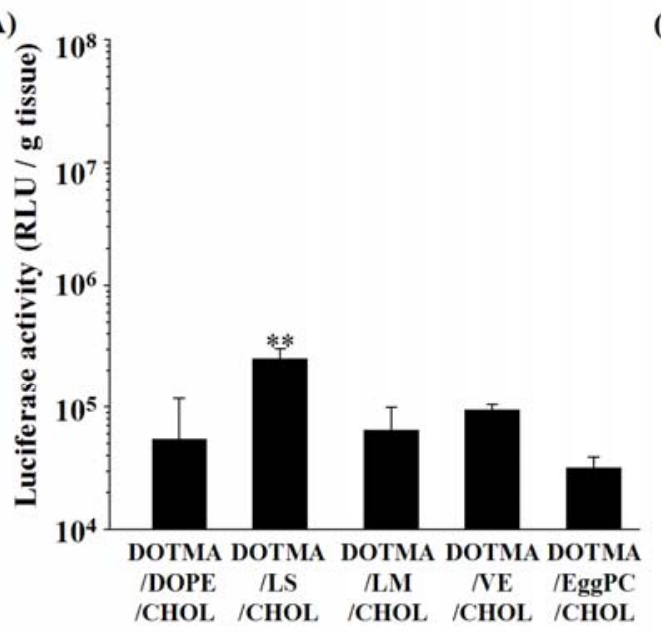

(C)

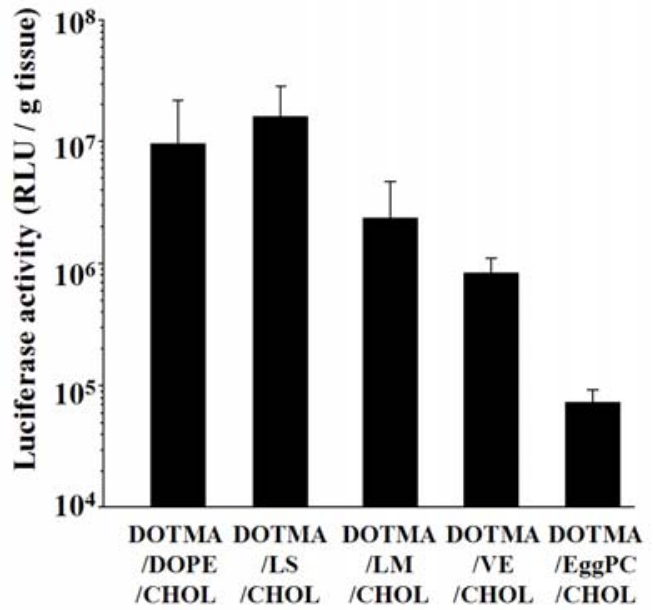

(E)

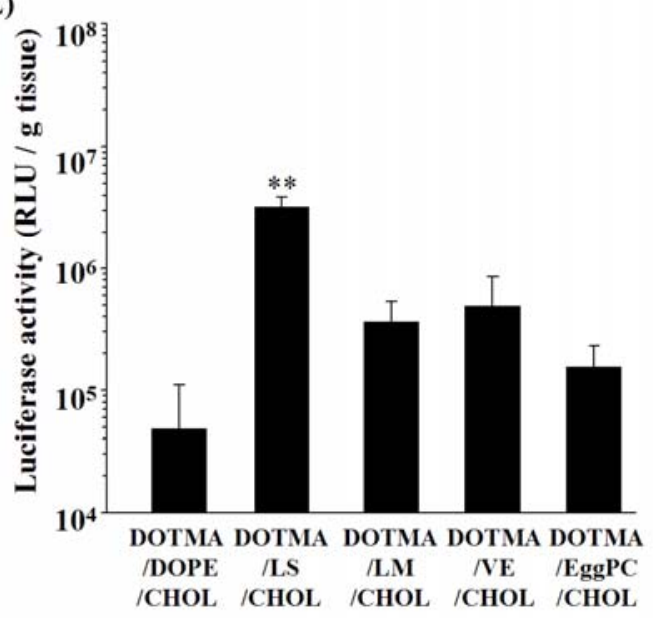

(B)

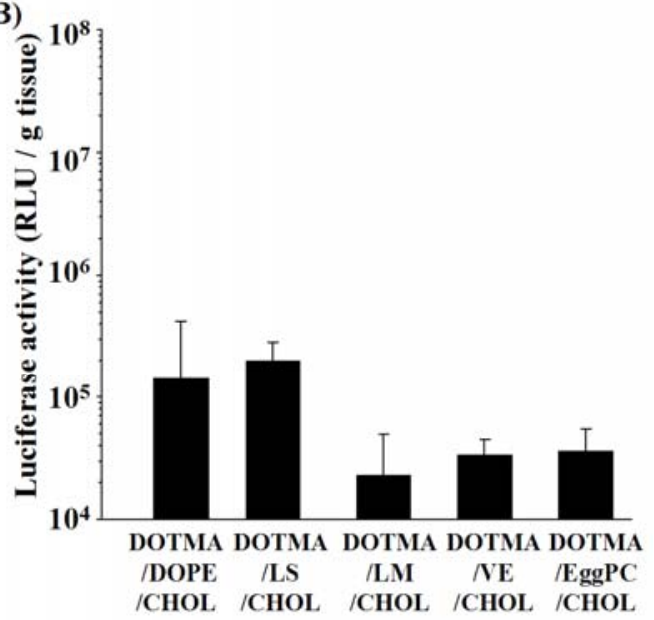

(D)

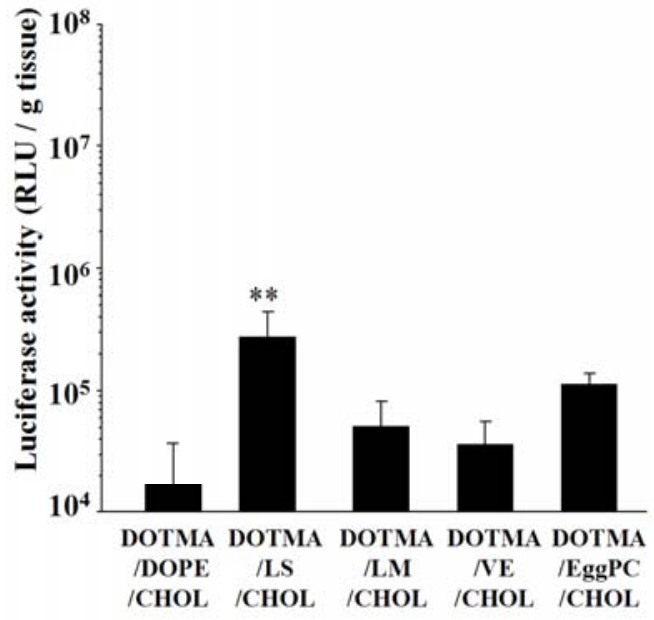

Figure 6. Luciferase gene expression of the various lipoplexes in vivo. Each lipoplexes was administrated intravenously to mice. After $6 \mathrm{~h}$, mice were sacrificed, and the luciferase activities of liver (A), kidney (B), spleen (C), heart (D), and lung (E) were determined. Each bar represents the mean + S.D. of three or six experiments. $\quad{ }^{* *} ; P<$ 0.01 vs DOTMA/DOPE/CHOL lipoplexes. 


\section{DISCUSSION}

In gene therapy, viral vectors have been utilized for many clinical trials due to their high ability to transfect. However, they have limitations in vivo such as wild-type reversion and immunogenicity. On the other hand, non-viral vectors including cationic liposomes and polymers have several advantages including non-immunogenicity, low acute toxicity, and flexibility to design a vehicle with well-defined structural and chemical properties to yield mass production $(17,18)$.

Cationic liposomes were known effective gene delivery vectors for human gene therapy $(19,20)$. To elevate effectivity of gene delivery, helper-lipids such as CHOL and DOPE were often added to cationic liposomes $(9,11,12)$. CHOL makes liposome membrane uncrushable, and the CHOL-containing liposomes had much higher expression as compared to other liposomes in vivo $(21,22)$. DOPE was reported to be a fusogenic lipid, and it made DNA possible to escape out of endosome by its conversion of a lamellar to a hexagonal phase (23).

In the present study, we newly exploited the alternative helper-lipids to DOPE among transdermal penetration enhancers. Transdermal penetration enhancers were known to make drugs much permeated into skin by disrupting the structure of stratum corneum lipid bilayers. DOPE was also reported to have a potential to enhance transdermal penetrations of the drug such as estradiol, progesterone, and propranolol (13).

LS, LM, VE, and EggPC have been reported to be effective transdermal penetration enhancers (24-30). We prepared several cationic liposomes containing these penetration enhancers with DOTMA and CHOL. Physicochemical characteristics such as particle sizes and $\zeta$-potentials of those liposomes were determined and are shown in Table 1. Transdermal penetration enhancers much affected the particle sizes and $\zeta$-potentials of the liposomes because of their interactions with membrane lipids. Especially, DOTMA/LS/CHOL liposomes significantly decreased $\zeta$-potentials by anionic charge of LS. The reduction of $\zeta$-potentials may decrease electrostatic objection and increase the particle sizes. These liposomes can formulate the electrostatic complex with pDNA, which was identified by gel electrophoresis (Figure 1). The pDNA was completely complexed with each liposomes, migrations of pDNA and pDNA bands not observed.

In vitro transfection efficiency and cell toxicity of theses lipoplexes were evaluated using human hepatoma cell line HepG2 in the presence/absence of FBS (Figures 2 and 3). The cationic liposomes containing DOPE were reported as effective gene delivery vehicles in vitro (31-33). Actually, the DOTMA/DOPE/CHOL lipoplexes had high transfection efficiency in HepG2 in the absence of FBS. DOTMA/LS/CHOL lipoplexes had significantly higher efficiency although DOTMA/LM, VE, EggPC/CHOL lipoplexes showed equivalent efficiency. The serum protein bound to the cationic lipoplexes electrostatically, and the lipoplexes has been reported to reduce their transfection efficiency in the presence of FBS (34, 35). The present lipoplexes induced the transfection efficiency in the presence of FBS. They may have the in vivo potential as gene delivery vectors. On the other hand, all lipoplexes showed cell toxicity on control in the absence of FBS. In the presence of FBS, DOTMA/LS/CHOL lipoplexes showed low cytotoxicity on control although DOTMA/DOPE/CHOL had a severe cytotoxicity.

We examined interaction of the lipoplexes to erythrocytes before in vivo experiments (Figure 4). In many studies, the cationic liposomes containing DOPE had reported to agglutinate erythrocytes (36, 37). The agglutinations are proposed to explain the discrepancies between in vitro and in vivo transfection results (38). A strong agglutination was observed in DOTMA/DOPE/CHOL lipoplexes although DOTMA/LS, VE, EggPC/CHOL lipoplexes did not show agglutination. A festinate hemolysis was found in DOTMA/LM/CHOL lipoplexes. Therefore, we quantitatively determined hemoglobin released by hemolysis and show it in Figure 5. The high hemolysis was confirmed in DOTMA/DOPE/CHOL lipoplexes and DOTMA/LM/CHOL lipoplexes. DOTMA/LS/CHOL, DOTMA/VE/CHOL, and DOTMA/EggPC/CHOL lipoplexes showed 
extremely lower hematotoxicity.

On the basis of these results, the in vivo transfection efficiency of the lipoplexes was examined in mice. The luciferase activities in the five organs such as liver, kidney, spleen, heart, and lung were determined at $6 \mathrm{~h}$ after each lipoplexes was administrated intravenously to mice (Figure 6). The luciferase activities of DOTMA/DOPE/CHOL lipoplexes were below $10^{5} \mathrm{RLU} / \mathrm{mg}$ protein in the liver, heart, and lung, but it was reported that luciferase activities of DOTMA/DOPE and DOTMA/CHOL lipoplexes were below $10^{3}$ and $10^{5}$ respectively in the lung even if the high charge ratio +2.24 (37). The lack of serum stability greatly limits their potential application of DOPE lipoplexes in vivo (39). DOTMA/DOPE was also reported to agglutinate with erythrocytes and be embolized in the lung capillary with erythrocytes (36). On the other hand, the lipoplexes containing LM, VE, and EggPC stacked up to DOTMA/DOPE/CHOL lipoplexes, but DOTMA/LS/CHOL lipoplexes showed significantly higher transfection efficiency than DOTMA/DOPE/CHOL lipoplexes in the liver, heart, and lung.

Generally, the complexes with strong positive charge show high gene expression because of their strong interaction with cell membranes. On the other hand, the strong positive charge on the complexes turns to aggregate with erythrocytes and serum protein, and eliminate rapidly in the blood (40). The large aggregates of the lipoplexes with erythrocytes were suggested to be embolized in the pulmonary vein and show high accumulations in the lung (36). However, the large aggregates may cause problems because of microinfarction, causing tissue ischemia and possible myocardial damage (41).

In the present study, we found that the lipoplexes containing LS had high transgene efficiency and low toxicity under in vitro and in vivo conditions. A low cationic charge of the lipoplexes containing LS decreased aggregation with erythrocytes and cytotoxicity. High gene expression of the lipoplexes containing LS may be caused by the fusogenic ability of LS. Although the particles sized more than 5 micrometer are known to accumulate in the lung, the slightly large particle of the lipoplexes containing LS might contribute to pulmonary delivery. Further studies about cellular internalization pathway, transcription efficiency, and cytosol dependent stability may be necessary to figure out the mechanism for their transgene efficiency.

\section{CONCLUSION}

It is concluded that several transdermal penetration enhancers may be available for alternative helper-lipids to DOPE in cationic liposomal vectors. DOTMA/LS/CHOL lipoplexes showed superior characteristics in comparison with DOTMA/DOPE/CHOL lipoplexes in in vitro transfection efficiency, cell toxicity, hematotoxicity, and in vivo transfection efficiency.

\section{ACKNOWLEDGMENTS}

The authors would like to thank Dr. Takuro Niidome for his assistance with $\zeta$-sizer Nano-ZS measurement.

\section{REFERENCES}

[1]. Edelstein, M.L., Abedi, M.R., Wixon J. Gene therapy clinical trials worldwide to 2007 -an update. J. Gene Med., 9: 833-842. 2007.

[2]. Gautam, A., Densmore, C.L., Melton, S., Golunski, E., Waldrep, J.C. Aerosol delivery of PEI-p53 complexes inhibits B16-F10 lung metastases through regulation of angiogenesis. Cancer Gene Ther., 9: 28-36. 2002.

[3]. Varmus, H. Retroviruses. Science, 240: 1427-1435. 1988.

[4]. Gao, X., Kim, K.S., Liu, D. Nonviral gene delivery: What we know and what is next. AAPS J. 9: E92-104. 2007.

[5]. Sorgi, F.L., Bhattacharya, S., Huang, L. Protamine sulfate enhances lipid-mediated gene transfer. Gene Ther., 4: 961-968. 1997.

[6]. Meyer, O., Kirpotin, D., Hong, K., Sternberg, B., Park, J.W., Woodle, M.C., Papahadjopoulos, D. Cationic liposomes coated with polyethylene glycol as carriers for oligonucleotides. J. Biol. 
Chem., 273: 15621-15627. 1998.

[7]. Zhdanov, R.I., Podobed, O.V., Vlassov, V.V. Cationic lipid-DNA complexes-lipoplexes for gene transfer and therapy. Bioelectrochemistry, 58: 53-64. 2002.

[8]. Felgner, P.L., Gadek, T.R., Holm, M., Roman, R., Chan, H.W., Wenz, M., Northrop, J.P., Ringold, G.M., Danielsen, M. Lipofection: a highly efficient, lipid-mediated DNA-transfection procedure. Proc. Natl. Acad. Sci. U.S.A., 84: 7413-7427. 1987.

[9]. Hong, K., Zheng, W., Baker, A., Papahadjopoulos, D. Stabilisation of cationic liposome/DNA complexes by polyamines and polyethylenglycol-phospholipid conjugates for efficient in vivo gene delivery. FEBS Lett., 414: 187-192. 1997.

[10]. Lasic, D.D. Liposomes in Gene Delivery, CRC Press, Boca Raton. 1997.

[11]. Farhood, H., Serbina, N., Huang, L. The role of dioleoylphosphatidylethanolamine in cationic liposome mediated gene transfer. Biochim. Biophys. Acta., 1235: 289-295. 1995.

[12]. Koltover, I., Salditt, T., Radler, J.O., Safinya, C.R. An inverted hexagonal phase of cationic liposome-DNA complexes related to DNA release and delivery. Science, 281: 78-81. 1998.

[13]. Kirjavainen, M., Mönkkönen, J., Saukkosaari, M., Valjakka-Koskela, R., Kiesvaara, J., Urtti, A. Phospholipids affect stratum corneum lipid bilayer fluidity and drug partitioning into the bilayers. J. Control. Release, 58: 207-214. 1999.

[14]. Kanikkannan, N., Kandimalla, K., Lamba S.S., Singh, M. Structure-activity Relationship of Chemical Penetration Enhancers in Transdermal Drug Delivery. Curr. Med. Chem., 6: 593-608. 2000.

[15]. Hadgraft, J. Skin deep. Eur. J. Pharm. Biopharm., 58: 291-299. 2004.

[16]. Kawakami, S., Yamamura, K., Mukai, T., Nishida, K., Nakamura, J., Sakaeda, T., Nakashima, M., Sasaki, H. Sustained ocular delivery of tilisolol to rabbits after topical administration or intravitreal injection of lipophilic prodrug incorporated in liposomes. J. Pharm. Pharmacol., 53: 1157-1161. 2001.

[17]. Tang, M.X. and Szoka, F.C. The influence of polymer structure on the interactions of cationic polymers with DNA and morphology of the resulting complexes. Gene Ther., 4: 823-832. 1997.

[18]. Mario, C.F. and Nigel, C.P. Major limitations in the use of cationic liposomes for DNA delivery. Int. J. Pharm., 162: 159-170. 1998.

[19]. Tsukamoto, M., Ochiya, T., Sugimura, S.T., Terada, M. Gene transfer and expression in progeny after intravenous DNA injection into pregnant mice. Nat. Genet., 9: 243-248. 1995.

[20]. Woodle, M.C. and Scaria, P. Cationic liposomes and nucleic acids. Curr. Opin. Colloid and Interface Sci., 6: 78-84. 2001.

[21]. Bennet, M.J., Nantz, M.H., Balasubramanian, R.P. Gruenert, D.C., Malone, R.W. Cholesterol enhances cationic liposome-mediated DNA transfection of human respiratory epithelial cells. Biosci. Rep., 15: 47-53. 1995.

[22]. Tempelton, N.S., Lasic, D.D., Frederik, P.M., Strey, H.H., Roberts, D.D., Palvakis, G.N. Improved DNA:liposomes complexes for increased systemic delivery and gene expression. Nat. Biotechnol., 15: 647-652. 1997.

[23]. Guo, X. and Szoka, F.C. Steric stabilization of fusogenic liposomes by a low $\mathrm{pH}$ sensitive PEG-diorthoester-lipid conjugate. Bioconjug. Chem., 12: 291-300. 2001.

[24]. Ahkong, Q.F., Fisher, D., Tampion, W., Lucy, J.A. The fusion of erythrocytes by fatty acids, esters, retinol, and $\alpha$-tocopherol. Biochem. J., 135: 147-155. 1973.

[25]. Trotta, M., Pattarino, F., Gasco, M.R. Influence of counter ions on the skin permeation of methotrexate from water-oil microemulsions. Pharm. Acta Helv., 71: 135-140. 1996.

[26]. Krishnaiah, Y.S., Satyanarayana, V., Bhaskar, P. Influence of limonene on the bioavailability of nicardipine hydrochloride from membrane-moderated transdermal therapeutic systems in human volunteers. Int. J. Pharm., 247: 91-102. 2002.

[27]. Paolino, D., Ventura, C.A., Nistico, S., Puglisi, G., Fresta, M. Lecithin microemulsions for the topical administration of ketoprofen: percutaneous adsorption through human skin and in vivo human skin tolerability. Int. J. Pharm., 244: 21-31. 2002.

[28]. Karande, P., Jain, A., Mitragotri, S. Discovery of 
transdermal penetration enhancers by high-throughput screening. Nat. Biothechnol., 22: 192-197. 2004.

[29]. Lim, P.F., Liu, X.Y., Kanga, L., Ho, P.C., Chan, Y.W., Chan, S.Y. Limonene GP1/PG organogel as a vehicle in transdermal delivery of haloperidol. Int. J. Pharm., 311: 157-164. 2006.

[30]. Karande, P., Jain, A., Arora, A., Ho, M.J., Mitragotri, S. Synergistic effects of chemical enhancers on skin permeability: A case study of sodium lauroylsarcosinate and sorbitan monolaurate. Eur. J. Pharm. Sci., 31: 1-7. 2007.

[31]. Hui, S.W., Langner, M., Zhao, Y.L., Hurley, E., Chan, K. The role of helper lipids in cationic liposome-mediated gene transfer. Biophys. J., 71: 590-599. 1996.

[32]. Mok, K.W. and Cullis, P.R. Structural and fusogenic properties of cationic liposomes in the presence of plasmid DNA. Biophys. J., 73: 2534-2545. 1997.

[33]. Simões, S., Slepushkin, V., Gaspar, R., de Lima, M.C., Düzgünes, N. Gene delivery by negatively charged ternary complexes of DNA, cationic liposomes and transferrin or fusigenic peptides. Gene Ther., 5: 955-964. 1998.

[34]. Xu L. Anchordoquy T.J. Cholesterol domains in cationic lipid/DNA complexes improve transfection. Biochim. Biophys. Acta., 10: 2177-2181. 2008.

[35]. Crook K., Stevenson B.J., Dubouchet M., Porteous D.J. Inclusion of cholesterol in DOTAP transfection complexes increases the delivery of DNA to cells in vitro in the presence of serum. Gene Ther., 5: 137-143. 1998.
[36]. Sakurai, F., Nishioka, T., Yamashita, F., Takakura, Y., Hashida, M. Effects of erythrocytes and serum proteins on lung accumulation of lipoplexes containing cholesterol or DOPE as a helper lipid in the single-pass rat lung perfusion system. Eur. J. Pharm. Biopharm., 52: 165-172. 2001.

[37]. Sakurai, F., Nishioka, T., Saito, H., Baba, T., Okuda, A., Matsumoto, O., Taga, T., Yamashita, F., Takakura Y., and Hashida, M. Interaction between DNA-cationic liposome complexes and erythrocytes is an important factor in systemic gene transfer via the intravenous route in mice: the role of the neutral helper lipid. Gene Ther., 8: 677-686. 2001.

[38]. Eliyahu, H., Servel, N., Domb, A.J., Barenholz, Y. Lipoplexes-induced hemagglutination: potential involvement in intravenous gene delivery. Gene Ther., 9: 850-858. 2002.

[39]. Gao, Y., Gu, W., Chen, L., Xu, Z., Li, Y. A multifunctional nano device as non-viral vector for gene delivery: In vitro characteristics and transfection. J. Control. Release, 118: 381-388. 2007.

[40]. Ogris M., Brunner S., Schuller S., Kircheis R., Wagner E. PEGylated DNA/transferrin-PEI complexes: reduced interaction with blood components, extended circulation in blood and potential for systemic gene delivery. Gene Ther., 6: 595-605. 1999.

[41]. Dass C.R. Cytotoxicity issues pertinent to lipoplex-mediated gene therapy in-vivo. J. Pharm. Pharmacol., 54: 593-601. 2002. 